\title{
Predictability and Management of OARs Toxicity in Patients with Prostate Cancer Treated with High-Dose Radiotherapy
}

\author{
Vincenzo Ravo ${ }^{1}$, Domenico Borrelli ${ }^{2}$, Sara Falivene ${ }^{3}$, Rossella Di Franco ${ }^{3}$, Matteo Muto ${ }^{2}$, \\ Angela Argenone ${ }^{1}$, Valentina Borzillo ${ }^{1}$, Fabrizio Cammarota ${ }^{1}$, Paolo Muto ${ }^{1}$ \\ ${ }^{1}$ Radiotherapy Unit, Istituto Nazionale per lo Studio e la Cura dei Tumori "Fondazione Giovanni Pascale"-IRCCS, Naples, Italy; \\ ${ }^{2}$ Radiotherapy Emicenter, Naples, Italy; ${ }^{3}$ Radiotherapy of Second University of Naples, Naples, Italy. \\ Email: sara.falivene@gmail.com
}

Received November $7^{\text {th }}, 2013$; revised November $27^{\text {th }}, 2013$; accepted December $4^{\text {th }}, 2013$

Copyright (C) 2013 Vincenzo Ravo et al. This is an open access article distributed under the Creative Commons Attribution License, which permits unrestricted use, distribution, and reproduction in any medium, provided the original work is properly cited. In accordance of the Creative Commons Attribution License all Copyrights (C) 2013 are reserved for SCIRP and the owner of the intellectual property Vincenzo Ravo et al. All Copyright (C) 2013 are guarded by law and by SCIRP as a guardian.

\begin{abstract}
Aim: To evaluate the predictability of toxicity analyzing the dose-volume histograms (DVHs) and to verify the effectiveness of preventive measures limiting side toxicity considering the evolution of the radiation techniques for prostate cancer treatment. Materials and Methods: 208 patients with localized prostate cancer were treated with exclusive radiotherapy until 73.8 Gy (group A) or 79.2 Gy (group B) with the dose escalation technique. Preventive measures to minimize the side effects were recommended in group B. Results: The assessment of genitourinary toxicity was similar while gastrointestinal toxicity was better in group B. Valuating the treatment plans, we found that most of the patients developing toxicity had "borderline" DVHs. Conclusion: Our analysis led to the establishment of a protocol for the management of patients with "border-line" DVH.
\end{abstract}

Keywords: Toxicity; Radiotherapy; Prostate Cancer; Prevention

\section{Introduction}

Prostate cancer is the most common male cancer in industrialized countries and the diagnosis is made more frequently in the initial stage. It plays an important role in public health and in oncological clinical practice. The therapeutic process for prostate cancer underwent a deep change both in surgical management and in medical approach and above all in radiation therapy (RT) treatment, reaching high levels of efficiency and improvement of quality of life (QoL) of patients (pts). The evolution of the radiation techniques has made it possible to conform radiation beams looking at the target and saving adjacent organs at risk (OARs) so this has allowed treatments in the so-called "dose escalation". The increased dose has enabled more and more curative treatments, resulting, at the same time, in an increase in side effects to OARs as rectum and bladder [1,2]. The primary aim of our study was to evaluate if it is possible to predict the toxicity to
OARs analyzing the dose-volume histograms (DVHs) in pts treated with RT alone for localized prostate cancer [3-8]. Secondary objective of our study was to evaluate the effectiveness of preventive measures to limit as much as possible gastrointestinal and urinary side effects.

\section{Materials and Methods}

From January to December 2010, 208 pts with localized prostate cancer were treated with exclusive RT. The patients mean age was 66 years (range 49 - 83) and the Gleason Score average was 7 (range 5 -9). All pts were treated with concomitant hormone therapy with bicalutamide alone or maximal androgen blockade (MAB). Pts that refused or could not receive these therapies are excluded from the study. All patients were subjected to TC scan using a personalized immobilization system (Vac-Lock). In order to obtain a better reproducibility of the daily treatment all pts had to drink $500 \mathrm{cc}$ of water 
(filled bladder) and to empty the rectum before the TC scan and then before each treatment session. On CT images processed with a system of treatment planning the contouring of the Target and OARs (bladder, rectum and femoral heads) were made; then the treatment plans were elaborated. The treatment plan was elaborated with the technique of "dose escalation" and it was divided into two phases:

- the first phase up to $66.6 \mathrm{~Gy}$ (1.8 Gy per fraction for 37 fractions);

- the second phase from $66.6 \mathrm{~Gy}$ to $73.8 \mathrm{~Gy}$ or $79,2 \mathrm{~Gy}$ (1.8 Gy per fraction for 4 or 7 fractions).

In the first phase prostate and seminal vesicles were considered as Clinical Target Volume (CTV, i.e. GTVGross Tumor Volume - plus the area of supposed microscopical local diffusion) while in the second time CTV was only comprehensive of the prostate gland volume. The Planning Target Volume (PTV) was obtained by adding a margin of $1 \mathrm{~cm}$ in all directions and only $0.5 \mathrm{~cm}$ posteriorly. The treatment plan was developed with 3D-Conformal technique (3DCRT) and the treatment was performed with a Linear Accelerator with multilamellar collimator. The dose was distributed through six or seven fields of $18 \mathrm{MV}$ photon. During the planning it was assessed the dose distribution to the PTV and to the organs at risk according the dose costraints reported in Table 1 and using the analysis of DVHs (Dose Volume Histograms). Patients (Ptz) were divided into two groups: the group A of 100 pts treated with a total dose of 73.8 Gy (1.8 Gy in 41 fractions) and the group B of 108 pts treated with a total dose of 79.2 Gy $(1.8$ Gy in 44 fractions). An important difference between the two groups was the adoption of preventive measures to minimize the side effects from RT in group B. In particular it was racommended a low-fiber diet and the use of lactic ferments symbiotic (probiotic-prebiotic) to prevent acute gastrointestinal toxicity, the intake of supplement based on D-mannose and Proctocyanide to prevent urinary toxicity. Before starting, the radiation oncologist may suggest a proper diet, with drastic reduction of fat correlating with a reduced release of bile salts and fewer episodes of diarrhea. Than the pharmacological aids such as antiinflammatory, anti-diarrheal, anticholinergics, and

Table 1. Constraints of dose for rectum, bladder, femoral heads.

\begin{tabular}{cc}
\hline & CONSTRAINTS of DOSE \\
\hline Rectum & $30 \% \mathrm{~V}<70 \mathrm{~Gy}-60 \% \mathrm{~V}<40 \mathrm{~Gy}$ \\
Bladder & $50 \% \mathrm{~V}<70 \mathrm{~Gy}$ \\
Femoral heads & $50 \% \mathrm{~V}<50 \mathrm{~Gy}$ \\
\hline
\end{tabular}

probiotics strengthing the antioxidant defense systems of the mucosal cells can be recommended. In the treatment of actinic cystitis anti-inflammatory drugs and/or antibiotics or supplements based on blueberry, hibiscus, Dmannose. Among the products used in a preventive way there are the proantocyanide and the D-mannose. For all pts was scheduled weekly medical examination in order to evaluate acute lower gastrointestinal toxicity and/or urinary toxicity using Acute Radiation Morbidity Scoring Criteria of Radiation Therapy Oncology Group (RTOG).

International Prostate symptom score (IPSS) questionnaire was administered every week allowing to obtain an early detection of the onset or increasing of any urinary disorders. The follow up visits were made at 45 days, 3 months, and then every 6 months for two years after the end of RT. During follow up it was focused on IPSS to observe the eventual variation of urinary disorders and was evaluated chronic toxicity using Late Radiation Morbidity Scoring Criteria of RTOG.

\section{Results}

In Group A it was observed:

- Acute toxicity: $2.2 \%$ of urinary incontinence, $4.4 \%$ of dysuria/strangury; $13.3 \%$ of proctitis, rectal bleeding $2.2 \%$ and $2.2 \%$ of diarrhea/rectal tenesmus;

- Chronic Toxicity: $2.2 \%$ of hematuria, $6.6 \%$ of proctitis, rectal bleeding of $4.4 \%$ and $4.4 \%$ of diarrhea/ tenesmus.

In Group B it was observed:

- Acute toxicity: $2.5 \%$ urinary retention, urinary incontinence of $0.6 \%, 4.3 \%$ of dysuria/strangury, $4.9 \%$ of proctitis, rectal bleeding $1.2 \%$ and $3.1 \%$ of diarrhea/rectal tenesmus;

- Chronic Toxicity: $1.8 \%$ of hematuria, $1.2 \%$ urinary retention, urinary incontinence $1.8 \%, 0.6 \%$ actinic cystitis, $6.7 \%$ of proctitis, rectal bleeding $1.8 \%$ and $1.8 \%$ of diarrhea/rectal tenesmus (Table 2).

In the group A, no one developed urinary toxicity G2, $\mathrm{G} 3$ or G4, while in the group B the acute toxicity was G2 in 4 pts without $\mathrm{G} 3$ or $\mathrm{G} 4$ cases. The late toxicity was $\mathrm{G} 2$ in 2 pts, G3 in 1 pt and G4 in none pts. In the group A the acute rectal toxicity was G2 in 2 pts but none had G3 or G4 toxicity in the same way the late rectal toxicity was G2 in 2 pts and none had G3 or G4 toxicity. In the group B 6 pts developed acute rectal toxicity G2 and none G3-G4 then the late rectal toxicity was G2 in 5 pts and G3 in 1 pts (Table 3).

Later, re-evaluating the treatment plans, we found that most of patients developing toxicity had DVHs that could be defined as "borderline", considering the respect of the dose constraints, into the limits of tolerance dose to OARs. In these cases the treatment was performed 
Table 2. The urinary and rectal toxicity observed in acute and chronic phases both in Group A and in Group B.

\begin{tabular}{|c|c|c|c|c|c|}
\hline & & & Group A ( $\%$ of patients) & & Group B ( $\%$ of patients) \\
\hline & & Hematuria & 0 & & 2.2 \\
\hline & & Urinary Retention & 0 & & 0 \\
\hline \multirow[t]{4}{*}{ Urinary Toxicity } & Acute & Urinary Incontinence & 2.2 & Chronic & 0 \\
\hline & & Dysuria/Strangury & 4.4 & & 0 \\
\hline & & Actinic Cystitis & 0 & & 0 \\
\hline & & Proctitis & 13.3 & & 6.6 \\
\hline \multirow[t]{5}{*}{ Gastrointestinal Toxicity } & Acute & Rectal Bleeding & 2.2 & Chronic & 4.4 \\
\hline & & Diarrhea/Rectal Tenesmus & 2.2 & & 4.4 \\
\hline & & & $\%$ & & $\%$ \\
\hline & & Hematuria & 0 & & 1.8 \\
\hline & & Urinary Retention & 2.5 & & 1.2 \\
\hline \multirow[t]{4}{*}{ Urinary Toxicity } & Acute & Urinary Incontinence & 0.6 & Chronic & 1.8 \\
\hline & & Dysuria/Strangury & 4.3 & & 0 \\
\hline & & Actinic Cystitis & 0 & & 0.6 \\
\hline & & Proctitis & 4.9 & & 6.7 \\
\hline \multirow[t]{2}{*}{ Gastrointestinal Toxicity } & Acute & Rectal Bleeding & 1.2 & Chronic & 1.8 \\
\hline & & Diarrhea/Rectal Tenesmus & 3.1 & & 1.8 \\
\hline
\end{tabular}

Table 3. Urinary and Gastrointestinal Toxicity observed in the two groups of patients.

\begin{tabular}{ccccc}
\hline \multirow{4}{*}{ Urinary and Gastrointestinal Toxicity } & \\
\multirow{2}{*}{ RTOG Urinary Acute } & G2 & G3 & G4 \\
& Group A & 0 & 0 & 0 \\
& Group B & 4 & 0 & 0 \\
\multirow{2}{*}{ RTOG Urinary Chronic } & Group A & 0 & 0 & 0 \\
& Group B & 2 & 1 & 0 \\
RTOG Gastrointestinal Acute & Group A & 2 & 0 & 0 \\
& Group B & 6 & 0 & 0 \\
& & G2 & G3 & G4 \\
RTOG Gastrointestinal & Group A & 2 & 0 & 0 \\
Chronic & Group B & 5 & 1 & 0 \\
\hline
\end{tabular}

without any change in the dose prescription and the "risk" of side effects were justified in the majority of cases by the particular anatomy of the patient (Figures 1(a), (b) and Figure 2(a)). But if it is true that there were a high probabilities that a "border-line" DVHs could produce genitourinary or rectal toxicities, the opposite could not be true: but we founded that not all cases of toxicity are associated with "borderline" DVH parameters (Figure 2(b)). This means that the toxicity appearance is not only linked to the dose of radiations absorbed by the rectum and the bladder, but that there are other factors that could influence acute toxicity. From the comparison between borderline DVH and toxicity according to the RTOG scale, we noticed the presence of borderline DVH in $70 \%$ of patients with G1 toxicity, in $85 \%$ of those with G2 toxicity, in $100 \%$ of those with G3 toxicity. This suggest that when we obtained a "borderline" DVH during planning it means that we can have the $85 \%$ chance that the patient will develop acute toxicity. In cases of toxicity non related to a borderline DVH, we have to consider other factors that could be: diet, concomitant use of medical therapy, difficulty of the patient to empty the bladder for a concomitant benign prostatic hypertrophy or for another impediment, individual sensitivity, variability of positioning for poor compliance of patient to the treatment, organ interfraction and intrafraction motion.

\section{Discussion}

In the clinical management of pts undergoing RT for prostate cancer, the use of supportive therapies is useful for a better management of local toxicity and to improve the QoL. The gastrointestinal tract can develop acute actinic enteritis and proctitis around the second week of treatment, or chronic forms over a period of between 18 months and 6 years after treatment [1,2]. Acute cystitis is a frequent complication of RT for prostate cancer in fact over $20 \%$ of pts develop a chronic cystitis even many years after RT, while $9 \%$ have a macroscopic hematuria that is often recurrent. 

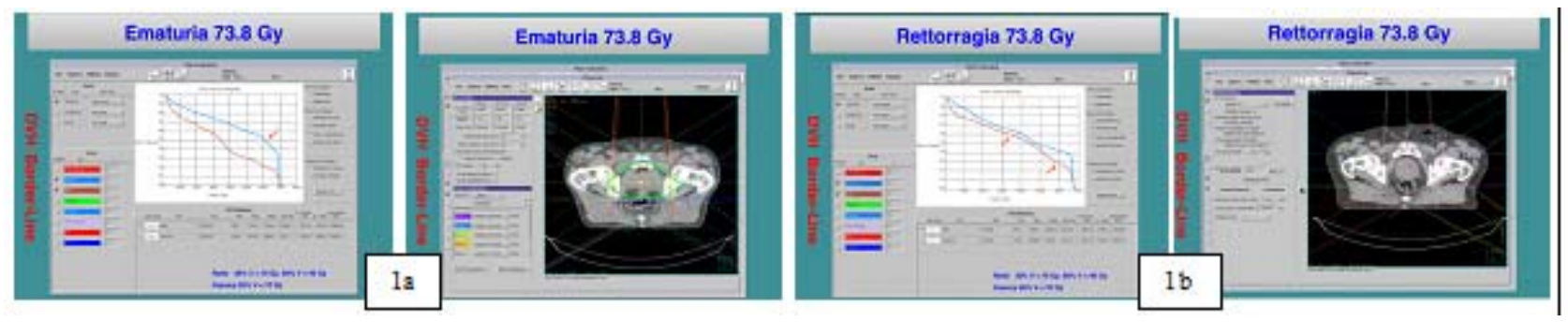

Figures 1. a-b: Bladder's and Rectal's DVHs in pts with higher risk of toxicity.

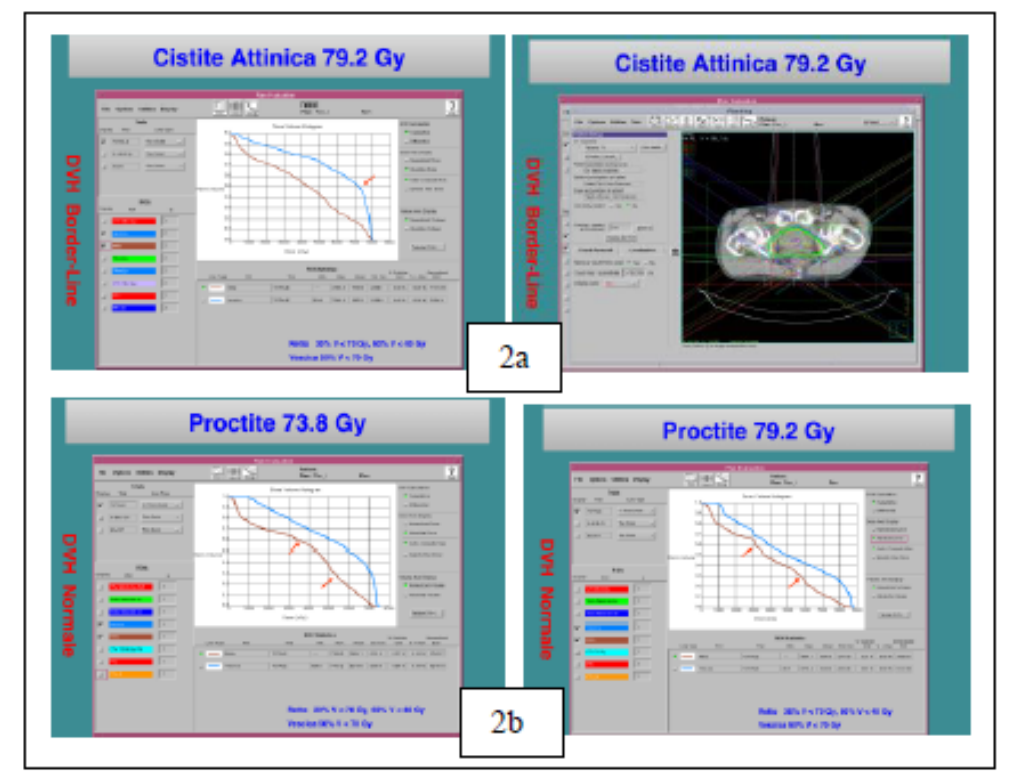

Figures 2. a-b: “Border-line” DVHs in pts with radiation cystitis and proctitis.

The onset of toxicity of high grades can become a dose-limiting condition invalidating the results of the RT so the evaluation of rectal DVH and bladder DVH is a crucial time in the planning phase [3-8].

Obviously the modern radiotherapy techniques have led to a significant reduction of the toxicity. The dose volume constraints are key starting points for the RT planning. For the treatment planning in $3 \mathrm{D}$, the values of $\mathrm{V} 50<50 \%$, V60 $<35 \%$, V65 $<25 \%$, V70 $<20 \%$, and $\mathrm{V} 75<15 \%$ of rectum should limit the Grade 2 rectal toxicity to $<15 \%$ and the probability of a Grade 3 rectal toxicity to $<10 \%$ for prescriptions up to 79.2 Gy in fractions of $1.8-2 \mathrm{~Gy}$. Higher doses have a greater impact on the probability of complications so we should try to minimize the V70 and V75 without compromising the coverage of the target [9]. It is known that the treatment in dose-escalation improves local and biochemical control in localized prostate cancer but it leads to an increase in late toxicity of normal tissues so it becomes essential the implementation of all the principals in order to minimize the effects side, especially in pts at higher risk of toxicity [10-13]. Obviously it is important to identify the patients who have a higher risk of toxicity. We know that in the treatment planning the anatomy of the patient is an important variable (Figures 1 and 2) in fact, in some cases it is unattainable reaching an acceptable DVH because of the anatomical variations. Various methods of evaluation of toxicity are available. Different scales for assessment the QoL have been developed and validated by measuring the impact of therapy after treatment of prostate cancer [14-17]. The scales of RTOG toxicity have been changed [18], and different gastrointestinal indicators have been used to characterize origin and clinical course of toxicity, for example, Peeters et al. [19] have characterized the gastrointestinal toxicity considering these indicators related to specific anatomical parameters and dose-volume. Post-void residual bladder volume was variable because bladder volume changed with the filling; the bladder may also move with the positioning, breathing, or filling of the intestine, so had to be considered bladder DVH obtained from the planning on centering CT. In the last decade the escalation of dose dispensed with conformant techniques, such as Intensity Modulated RT (IMRT), allowed to deliver high doses 
(>70 Gy) to prostate, so also to the lower portion of the bladder.

With these techniques, the upper part of the bladder is outside the field of treatment, but the region of the trigone may receive the same dose of target. Some studies have shown an association between the dose to the prostate and acute or chronic genitourinary toxicity. Zelefsky reported a cumulative incidence of genitourinary toxicity at 10 years from the end of RT to prostate that was $20 \%$ after $81 \mathrm{~Gy}$ in pts treated with IMRT compared with $12 \%$ in non-IMRT pts treated at lower doses. However, only $3 \%$ of the entire cohort had developed a genitourinary toxicity G3, while it was not observed toxicities G4 [20].

In our experience, the use of preventive measures in pts at high risk of toxicities predictable by DVHs examination allowed us to greatly reduce the side effects in pts treated with higher doses. The first procedure adopted was to prescribe a therapy with Bicalutamide or maximal androgen blockade (MAB) prior to the start of RT. This treatment was conducted for a minimum of two months according the AiroPros 01 - 02 study that showed a protective effect of hormonal therapy on $\mathrm{G}>2$ acute gastrointestinal toxicity $[21,22]$.

To prevent gastrointestinal toxicities we have suggested a diet low in fiber, to fill bladder before each RT session in order to keep away the bowel from the treatment field, the use of rectal gel made of hyaluronic acid to increase the trophism of the rectal mucosa, the administration of probiotics (lactic ferments such as Bac. Coagulans, St. thermophilus, Lb. Rhamnosus, Lb. Subtilis, Saccharomyces Boulardii) and prebiotics (inulin) of up to 30 days RT. Probiotics have a regulating effect on the epithelial barrier in different ways: a direct action on the epithelium bowel increasing the synthesis and secretion of mucin by goblet cells; competition with the pathogens and commensal; improvement the stability of the tight-junctions reducing epithelial permeability to pathogens and their products; down-regulation of nuclear genes that encode for pro-inflammatory cytokines; downregulation of apoptosis maintaining a balance between proliferation and cell degeneration; positive effects on immunity increasing the local proliferation of IgA-secreting cells in the lamina propria and promoting the secretion of IgA in the intraluminally mucous layer. The inulin is a non-digestible food component that induces selective stimulation of the growth and of activity of one or more genera/bacterial species of intestinal microbiota with beneficial properties for the host [23]. To prevent genitourinary toxicities we had administered proantocyanide and D-mannose based preparations. Proantocyanide based substance contrast the adhesion of Bacterium Coli to the mucosa of the urinary tract facilitating their expulsion in the urine. The D-mannose based drug arrives in the urinary canal and binds to the walls and to the bacteria that may have started the colonization determining the gap thanks to the sticky structure so it promotes their expulsion with urination.

We have recommended the adoption of preventive measures illustrated throughout the period of time between the TC centering and a month after the end of the RT. Comparing the two groups of pts, we noticed that acute urinary toxicity was comparable except for the appearance of a case of urinary retention in the group B; instead chronic urinary toxicity was registered only in group B with the appearance of 1 case of actinic cystitis, 2 cases of urinary retention and 3 of urinary incontinence. Both acute than chronic rectal toxicity, paradoxically, was higher in pts of Group A, with appearance of proctitis and rectal bleeding. We have attributed this paradox to the absence of prescription of preventive measures in Group A. Therefore we retain that is useful to adopt those aids limiting the toxicity of RT in all pts candidates for pelvic RT.

\section{Conclusions}

Our study has shown that it is possible to predict the toxicity to OARs analyzing the dose-volume histograms (DVHs) in patients treated with RT for localized prostate cancer. Preventive measures can help limit gastrointestinal and urinary side effects. Our analysis led to the establishment of a protocol for the management of patients with prostate so-called "border-line" DVH with the plan of:

- more aggressive preventive treatments, such as prescription of lactic ferments and probiotics since the simulation session and up to 30 days after the end of RT, the prescription of a diet lacking in fiber and products containing D-mannose and proantocyanide that would restrict the risk of cystitis;

- weekly clinical visit during RT period;

- prescription uroflowmetry and bladder ultrasound with evaluation of post-void residual before RT;

- visits and follow-up examinations more frequently.

\section{REFERENCES}

[1] M. M. Bismar and F. A. Sinicrope, "Radiation Enteritis," Current Gastroenterology Reports, Vol. 4, No. 5, 2002, pp. 361-365. http://dx.doi.org/10.1007/s11894-002-0005-3

[2] K. Leiper and A. I. Morris, "Treatment of Radiation Proctitis," Clinical Oncology (Royal College of Radiologists), Vol. 19, No. 9, 2007, pp. 724-729. http://dx.doi.org/10.1016/j.clon.2007.07.008

[3] E. H. Huang, A. Pollack, L. Levy, G. Starkschall, L. Dong, I. Rosen and D. A. Kuban, "Late Rectal Toxicity: Dose-Volume Effects of Conformal Radiotherapy for 
Prostate Cancer," International Journal of Radiation Oncology, Biology, Physics, Vol. 54, No. 5, 2002, pp. 13141321.

http://dx.doi.org/10.1016/S0360-3016(02)03742-2

[4] R. Miralbell, D. Taussky, O. Rinaldi, A. Lomax, S. Canales, L. Escude, P. Nouet, O. Ozsoy and M. Rouzaud, "Influence of Rectal Volume Changes during Radiotherapy for Prostate Cancer: A Predictive Model for Mild-ToModerate Late Rectal Toxicity," International Journal of Radiation Oncology, Biology, Physics, Vol. 57, No. 5, 2003, pp. 1280-1284.

http://dx.doi.org/10.1016/S0360-3016(03)00749-1

[5] S. T. Peeters, W. D. Heemsbergen, W. L. van Putten, A. Slot, H. Tabak, J. W. Mens, J. V. Lebesque and P. C. Koper, "Acute and Late Complications after Radiotherapy for Prostate Cancer: Results of a Multicenter Randomized Trial Comparing 68 Gy to 78 Gy," International Journal of Radiation Oncology, Biology, Physics, Vol. 61, No. 4, 2005, pp. 1019-1034. http://dx.doi.org/10.1016/j.ijrobp.2004.07.715

[6] J. J. Nuyttens, S. Milito, P. F. Rust, A. T. Turrisi, "DoseVolume Relationship for Acute Side Effects during High Dose Conformal Radiotherapy for Prostate Cancer," Radiotherapy \& Oncology, Vol. 64, No. 2, 2002, pp. 209214. http://dx.doi.org/10.1016/S0167-8140(02)00185-8

[7] C. Fiorino, V. Vavassori, G. Sanguineti, C. Bianchi, G. M. Cattaneo, A. Piazzolla, C. Cozzarini, "Rectum Contouring Variability in Patients Treated for Prostate Cancer: Impact on Rectum Dose-Volume Histograms and Normal Tissue Complication Probability," Radiotherapy \& Oncology, Vol. 63, No. 3, 2002, pp. 249-255. http://dx.doi.org/10.1016/S0167-8140(01)00469-8

[8] J. J. Tuech, V. Chaudron, V. Thoma, J. C. Ollier, V. Tassetti, D. Duval and J. F. Rodier, "Prevention of Radiation Enteritis by Intrapelvic Breast Prosthesis," European Journal of Surgical Oncology, Vol. 30, No. 8, 2004, pp. 900-904. http://dx.doi.org/10.1016/j.ejso.2004.06.012

[9] A. L. Zietman, M. L. Desilvio, J. D. Slater, C. J. Rossi Jr., D. W. Miller, J. A. Adams, W. U. Shipley, "Comparison of Conventional-Dose vs High-Dose Conformal Radiation Therapy in Clinically Localized Adenocarcinoma of the Prostate: A Randomized Controlled Trial," JAMA, Vol. 294, No. 10, 2005, pp. 1233-1239. http://dx.doi.org/10.1001/jama.294.10.1233

[10] D. P. Dearnaley, M. R. Sydes, J. D. Graham, E. G. Aird, D. Bottomley, R. A. Cowan, R. A. Huddart, C. C. Jose, J. H. Matthews, J. Millar, A. R. Moore, R. C. Morgan, J. M. Russell, C. D. Scrase, R. J. Stephens, I. Syndikus, M. K. Parmar and RT01 Collaborators "Escalated-Dose versus Standard-Dose Conformal Radiotherapy in Prostate Cancer: First Results from the MRC RT01 Randomised Controlled Trial," The Lancet Oncology, Vol. 8, No. 6, 2007, pp. 475-487. http://dx.doi.org/10.1016/S1470-2045(07)70143-2

[11] I. Syndikus, R. C. Morgan, M. R. Sydes, J. D. Graham, and D. P. Dearnaley, "Late Gastrointestinal Toxicity after Doseescalated Conformal Radiotherapy for Early Prostate Cancer: Results from the UK Medical Research Council RT01 trial (ISRCTN47772397)," International Journal of
Radiation Oncology Biology Physics, Vol. 77, No. 3, 2010, pp. 773-783, http://dx.doi.org/10.1016/j.ijrobp.2009.05.052

[12] A. Al-Mamgani, W. L. van Putten, W. D. Heemsbergen, van G. J. Leenders, A. Slot, M. F. Dielwart, L. Incrocci and J. V. Lebesque, "Update of Dutch Multicenter DoseEscalation Trial of Radiotherapy for Localized Prostate Cancer," International Journal of Radiation Oncology, Biology, Physics, Vol. 72, No. 4, 2008, pp. 980-988. http://dx.doi.org/10.1016/j.ijrobp.2008.02.073

[13] A. Hille, N. Töws, H. Schmidberger and C. F. Hess, "A Prospective Three-Dimensional Analysis about the Impact of Differences in the Clinical Target Volume in Prostate Cancer Irradiation on Normal-Tissue Exposure. A Potential for Increasing the Benefit/Risk Ratio," Strahlentherapie und Onkologie, Vol. 181, No. 12, 2005, pp.

789-795. http://dx.doi.org/10.1007/s00066-005-1452-1

[14] R. Sauer, H. Becker, W. Hohenberger, C. Rödel, C. Wittekind, R. Fietkau, P. Martus, J. Tschmelitsch, E. Hager, C. F. Hess, J. H. Karstens, T. Liersch, H. Schmidberger and R. Raab, "German Rectal Cancer Study Group-Preoperative versus Postoperative Chemoradiotherapy for Rectal Cancer," The New England Journal of Medicine, Vol. 351, No. 17, 2004, pp. 1731-1740. http://dx.doi.org/10.1056/NEJMoa040694

[15] M. S. Litwin, R. D. Hays, A. Fink, P. A. Ganz, B. Leake, R. H. Brook, "The UCLA Prostate Cancer Index: Development, Reliability, and Validity of a Health-Related Quality of Life Measure," Medical Care, Vol. 36, No. 7, 1998, pp. 1002-1012. http://dx.doi.org/10.1097/00005650-199807000-00007

[16] J. T. Wei, R. L. Dunn, M. S. Litwin, H. M. Sandler and M. G. Sanda, "Development and Validation of the Expanded Prostate Cancer Index Composite (EPIC) for Comprehensive Assessment of Health-Related Quality of Life in Men with Prostate Cancer," Urology, Vol. 56, No. 6, 2000, pp. 899-905. http://dx.doi.org/10.1016/S0090-4295(00)00858-X

[17] S. T. Peeters, W. D. Heemsbergen, W. L. van Putten, A. Slot, H. Tabak, J. W. Mens, J. V. Lebesque and P. C. Koper, "Acute and Late Complications after Radiotherapy for Prostate Cancer: Results of a Multicenter Randomized Trial Comparing 68 Gy to 78 Gy," International Journal of Radiation Oncology, Biology, Physics, Vol. 61, No. 4, 2005, pp. 1019-1034.

http://dx.doi.org/10.1016/j.ijrobp.2004.07.715

[18] S. T. Peeters, M. S. Hoogeman, W. D. Heemsbergen, A. A. Hart, P. C. Koper and J. V. Lebesque, " Rectal Bleeding, Fecal Incontinence, and High Stool Frequency after Conformal Radiotherapy for Prostate Cancer: Normal Tissue Complication Probability Modeling," International Journal of Radiation Oncology, Biology, Physics, Vol. 66, No. 1, 2006, pp. 11-19. http://dx.doi.org/10.1016/j.ijrobp.2006.03.034

[19] M. J. Zelefsky, E. Aschkenasy, S. Kelsen and S. A. Leibel, "Tolerance and Early Outcome Results of Postprostatectomy Three-Dimensional Conformal Radiotherapy," International Journal of Radiation Oncology, Biol- 
ogy, Physics, Vol. 39, No. 2, 1997, pp. 327-333. http://dx.doi.org/10.1016/S0360-3016(97)00056-4

[20] C. L. Ohland and W. K. Macnaughton, "Probiotic Bacteria and Intestinal Epithelial Barrier Function," Gastrointestinal and Liver Physiology, Vol. 298, No. 6, 2010, pp. G807-819.

http://dx.doi.org/10.1152/ajpgi.00243.2009

[21] R. Valdagni, T. Rancati, C. Fiorino, G. Fellin, A. Magli, M. Baccolini, C. Bianchi, E. Cagna, C. Greco, F. A. Mauro, A. F. Monti, F. Munoz, M. Stasi, P. Franzone and V. Vavassori, "Development of a Set of Nomograms to Predict Acute Lower Gastrointestinal Toxicity for Prostate Cancer 3D-CRT," International Journal of Radiation Oncology, Biology, Physics, Vol. 71, No. 4, 2008 pp. 1065-1073. http://dx.doi.org/10.1016/j.ijrobp.2007.11.037

[22] V. Vavassori, C. Fiorino, T. Rancati, A. Magli, G. Fellin, M. Baccolini, C. Bianchi, E. Cagna, F. A. Mauro, A. F.
Monti, F. Munoz, M. Stasi, P. Franzone and R. Valdagni "Predictors for Rectal and Intestinal Acute Toxicities during Prostate Cancer High-Dose 3D-CRT: Results of a Prospective Multicenter Study," International Journal of Radiation Oncology, Biology, Physics, Vol. 67, No. 5, 2007, pp. 1401-1410.

http://dx.doi.org/10.1016/j.ijrobp.2006.10.040

[23] M. Roberfroid, G. R. Gibson, L. Hoyles, A. L. McCartney, R. Rastall, I. Rowland, D. Wolvers, B. Watzl, H. Szajewska, B. Stahl, F. Guarner, F. Respondek, K. Whelan, V. Coxam, M. J. Davicco, L. Léotoing, Y. Wittrant, N. M. Delzenne, P. D. Cani, A. M. Neyrinck and A. Meheust, "Prebiotic Effects: Metabolic and Health Benefits," British Journal of Nutrition, Vol. 104, Suppl. 2, 2010, pp. S1-63. http://dx.doi.org/10.1017/S0007114510003363 